\title{
Research on Dynamic Clustering Routing Considering Node Load for Wireless Sensor Networks*
}

\author{
Yi Sun, Can Cui, Shanshan Ke, Jun Lu \\ School of Electrical and Electronic Engineering, North China Electric Power University, Beijing, China \\ Email: sy@ncepu.edu.cn, cc52112345@163.com, keshanshan89@126.com, lujun@ncepu.edu.cn
}

Received May 2013

\begin{abstract}
Aiming at the problem that node load is rarely considered in existing clustering routing algorithm for Wireless Sensor Networks (WSNs), a dynamic clustering routing algorithm for WSN is presented in this paper called DCRCL (Dynamic Clustering Routing Considering Load). This algorithm is comprised of three phases including cluster head (CH) selection, cluster setup and inter-cluster routing. First, the $\mathrm{CHs}$ are selected based on residual energy and node load. Then the non- $\mathrm{CH}$ nodes choose a cluster by comparing the cost function of its neighbor $\mathrm{CHs}$. At last, each $\mathrm{CH}$ communicates with base station by using multi-hop communication. The simulation results show that comparing with the existing one, the techniques life cycle and date volume of the network are increased by 30.7 percent and 29.8 percent respectively by using the proposed algorithm DCRCL.
\end{abstract}

Keywords: Wireless Sensor Network; Dynamic Routing; Clustering Algorithm; Node Load

\section{Introduction}

As Wireless Sensor Network (WSN) is a kind of energy constrained multi-hop self-organizing networks, how to save energy and maximize life cycle is the core problem of WSN [1-3]. In cluster based WSN, the network is divided into distinct clusters with a single leader called cluster head (CH). CHs are either selected among normal senor nodes or in some case some high energy nodes called gateways which are deployed as $\mathrm{CHs}$ [4]. CHs transmit all the local data from normal nodes to base station (BS), so that normal nodes use less energy while $\mathrm{CHs}$ consume more. To prevent the death of $\mathrm{CHs}$ from extra load, the $\mathrm{CH}$ rotation mechanism is used to equilibrium energy consumption of the network by changing CHs dynamically in existing algorithm called LEACH [5]. However, the main disadvantage of LEACH is that a sensor node with very low energy may be selected as a $\mathrm{CH}$ and the $\mathrm{CHs}$ send the packet to BS directly in single hop communication. Thus, this method increases the energy consumption of the $\mathrm{CHs}$ and reduces the network life cycle.

A large number of algorithms have been developed to improve LEACH namely PEGASIS [6], HEED [7] etc. Compared to LEACH, PEGASIS improves network lifetime, but its data delay is significantly high and it is un-

*The work was supported by a grant from the National Science and Technology Major Project of China (No.2010ZX03006-005-01). suitable for large-sized networks. The HEED periodically selects $\mathrm{CHs}$ based on the node's residual energy and proximity measure of the neighbor nodes or node degree. In MRPUC [8], the authors design multi-hop routing and unequal clustering algorithm using residual energies of all sensor nodes and distance between sensor nodes to the BS to extend network lifetime. Although these algorithms can improve the network life cycle, the load problem is not considered. As a result all the data will be transmitted to the BS, the nearer from the node to the BS, the higher load it will have. Higher load means forwarding data more frequently, and consuming more energy. What's more, unbalanced load also leads to unbalanced energy consumption and the decrease of network life cycle.

In this paper, we propose a dynamic clustering routing algorithm which considers the load problem. This algorithm contains three phases namely $\mathrm{CH}$ selection, cluster setup and inter-cluster routing. In Section 2, these phases are introduced in detail. Simulation results are given in Section 3 followed by the conclusion in Section 4 .

\section{DCRCL Algorithm Description}

\subsection{Cluster Head Selection}

The general factors considered in $\mathrm{CH}$ selection are node residual energy, distance from $\mathrm{CH}$ to $\mathrm{BS}$, distribution of $\mathrm{CHs}$, communication cost within the cluster and so on. In DCRCL, the threshold function is optimized by consi- 
dering residual energy and node load.

In $\mathrm{CH}$ selection phase, every sensor node is given a random number from 0 to 1 . The node will be candidate $\mathrm{CH}$ if the random number is less than the threshold. The optimized threshold function is shown as follow:

$$
T(n)=\left\{\begin{array}{l}
\frac{p}{1-p[r \bmod (1 / p)]} \times\left[\lambda_{1} \frac{E_{\text {res }}}{E_{\text {ini }}}+\lambda_{2}\left(1-\frac{\eta}{k}\right)\right], n \in G \\
0
\end{array}\right.
$$

Where, $p$ is the probability of a node chosen to be $\mathrm{CH}$, $r$ is the present round, $G$ is a gather of nodes which were not chosen to be $\mathrm{CH}$ in the past $1 / p$ round. $E_{\text {res }}$ and $E_{\text {ini }}$ mean the residual energy and initial energy of nodes, $\eta$ is load factor and $k$ is normalization coefficient, $\lambda_{1}$ and $\lambda_{2}$ are weight coefficients.

The optimized function retains the advantage in LEACH that $\mathrm{CHs}$ are rotated by increasing the probability of being candidate $\mathrm{CH}$ for unselected nodes. At the same time, it balances energy and load to make the nodes with higher residual energy and lower load easier to be candidate $\mathrm{CH}$.

In the multi-hop network with a single BS, the load of $\mathrm{CH}$ contains not only the communication with nodes inside, also the channel utilize when $\mathrm{CH}$ transmit data from other CHs to BS $[9,10]$. The load considered in this paper is the latter.

As shown in Figure 1, the load of node A is the data comes from the nodes in dash area, indicated by $\eta$. In other words

$$
\eta=n \times q \times p k_{\text {_tize }}
$$

Where, $n$ is the node number in dash area, $q$ is the probability that a node transmit data in every moment, $p k \_s i z e$ is the size of every packet which is a same value for every node in the network. Suppose that nodes are distributed uniform in the network, so

$$
n=\frac{S_{0}}{S} \times N
$$

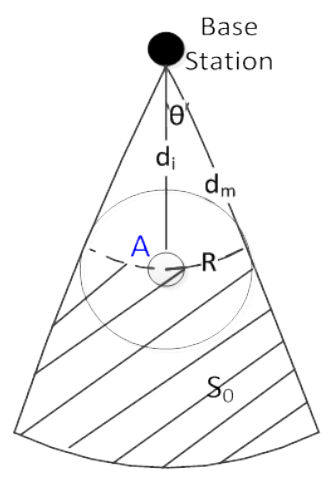

Figure 1. Node Load Model.
Where, $N$ is total node number, $S_{0}$ is the coverage of the dash area and $S$ is total coverage of the network.

On the base of the distance from A to BS namely $d_{i}$, communication radius $R$ and network range $d_{m}$, we can get

$$
\begin{gathered}
S_{0}=2 \theta \times \pi \times\left(d_{m}{ }^{2}-d_{i}{ }^{2}\right) \\
S=\pi d_{m}{ }^{2} \\
\theta=\arcsin \frac{R}{d_{i}}
\end{gathered}
$$

So

$$
\eta=2 N q \times \frac{\operatorname{arcsinR} / \mathrm{d}_{i} \times\left(\mathrm{d}_{\mathrm{m}}{ }^{2}-\mathrm{d}_{i}{ }^{2}\right)}{\mathrm{d}_{\mathrm{m}}{ }^{2}} \times p k \text { size }
$$

It is observed that node load is related to communication radius and the distance from node to BS.

\subsection{Cluster Setup}

In the cluster setup phase of DCRCL, CH competitive mechanism is introduced to avoid the unbalanced distribution of CHs in LEACH. After being a candidate $\mathrm{CH}$, a node competes with other candidates in its communication range $R$, the one who has most residual energy is the winner. $R$ is given as follow

$$
\mathrm{R}=\left(1+\alpha_{1} \frac{d_{i}}{d_{m}}+\alpha_{2} \frac{E_{\text {res }}}{E_{\text {ini }}}\right) \times R_{\text {min }}
$$

where, $d_{i}, d_{m}, E_{\text {res }}, E_{\text {ini }}$ are defined above. $R_{\min }$ is the minimum competition radius, $\alpha_{1}$ and $\alpha_{2}$ are weight coefficients and $\alpha_{1}+\alpha_{2}=1$.

Equation (7) shows that competition radius of $\mathrm{CH}$ and nodes in the cluster will decrease when the $\mathrm{CH}$ is nearer to BS and owns less residual energy. Considering that further CHs need close ones to transmit their data, the close CHs will have enough energy and free load to ensure the stability of the network in this way.

By Equation (7), it is known that $R \propto d_{i}$.

So $\theta$ can be approximated to a fixed value. Fused with Equation (5), we can see that

$$
k=2 \mathrm{Nq} \theta \times p k_{-} \text {size }
$$

Then, the selected $\mathrm{CHs}$ broadcast cluster request signal to the nodes in their communication range. Normal nodes choose which cluster to join in by using energy cost function shown as follow

$$
f(i, j)=\beta_{1} \frac{d_{(i, j)}}{E_{i}}+\beta_{2} \frac{d_{j}}{E_{j}}
$$

where, $d(i, j)$ is the distance from node $i$ to $\mathrm{CH}_{j}, d j$ is the distance from $\mathrm{CH}_{j}$ to $\mathrm{BS}, E_{i}$ and $E_{j}$ mean the residual energy of node $i$ and $\mathrm{CH}_{j}$. $\beta_{1}$ and $\beta_{2}$ are weight coefficients and $\beta_{1}+\beta_{2}=1$. 
As the Equation (8) shows, normal nodes prefer to cooperate with the $\mathrm{CH}$ which is closer to BS and owns more residual energy. The choose process is shown in Figure 2.

\subsection{Inter-Cluster Routing}

The model that data is transmitted directly from $\mathrm{CHs}$ to $\mathrm{BS}$, as known in LEACH, usually results in short life cycles of CHs and the network. In the proposed algorithm DCRCL, an energy balanced multi-hop communication model is established to use network energy more efficiently. The attribute equilibrium function for intercluster routing contains several factors namely residual energy, $\mathrm{CH}$ load and inside node number. The specific equation is as follows

$$
D_{E B i}=\omega_{1} N_{E i}+\omega_{2} N_{L i}+\omega_{3} N_{C i}
$$

where, $N_{E i}, N_{L i}, N_{C i}$ are uniformed $\mathrm{CH}$ residual energy, $\mathrm{CH}$ load and inside node number. $\omega_{1}, \omega_{2}$ and $\omega_{3}$ are weight coefficients and $\omega_{1}+\omega_{2}+\omega_{3}=1$.

$\mathrm{CHs}$ send routing request signal RREQ to the neighbor $\mathrm{CH}$ which has higher attribute equilibrium value than the average of all neighbors by computing the equilibrium function. At the same time, the link information in RREQ is updated. The information will be used by the destination node to choose a best link and send routing reply signal RREP, as shown in Figure 3.

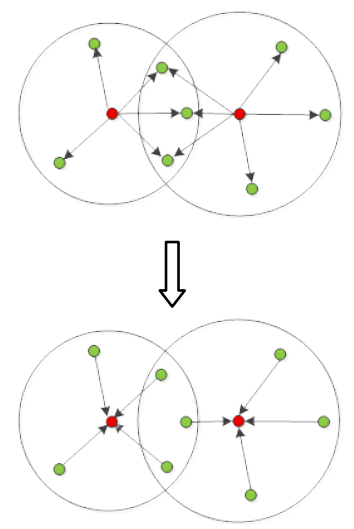

Figure 2. Cluster Setup Process.

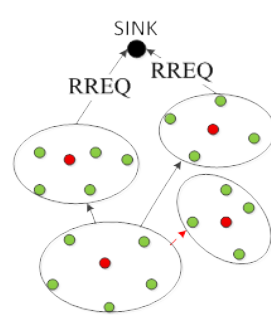

(a)

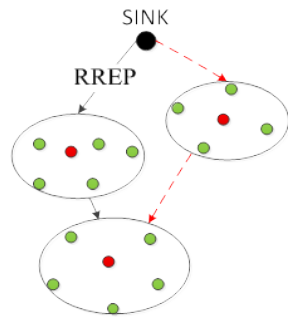

(b)
Figure 3. (a) Routing Request; (b) Routing Reply. Intercluster Routing Process.
When the inter-cluster routing is established, CHs record the link and compute the optimized transmission power according to the distance to next hop. The link will be used abidingly until next $\mathrm{CH}$ selection phase comes.

\section{Experimental Results}

Extensive experiments are performed for the proposed algorithm on MATLAB7.0 with the following experimental set up. All sensor nodes are distributed random in a $200 \mathrm{~m} \times 200 \mathrm{~m}$ area, all nodes are immobile with the same initial energy 0.1J. Nodes in cluster use TDMA mechanism on MAC layer and the communication mode is two-way. Time cycle of simulation is 300 rounds. For the sake of comparison, we also run the $\mathrm{LEACH}$ and AODV.

In Figure 4, the node number is 200 and the size of packet is 500 bit. We sampled the results every 15 rounds. As shown in Figure 4(a) that in the case of DCRCL, all nodes are inactive after 200 rounds, the network life cycle is increased by 566.7 percent and 33.3 percent compared with 30 rounds of AODV and 150 rounds of LEACH. Figure 4(b) shows that the data received by BS in DCRCL is 42.8 percent more than LEACH.

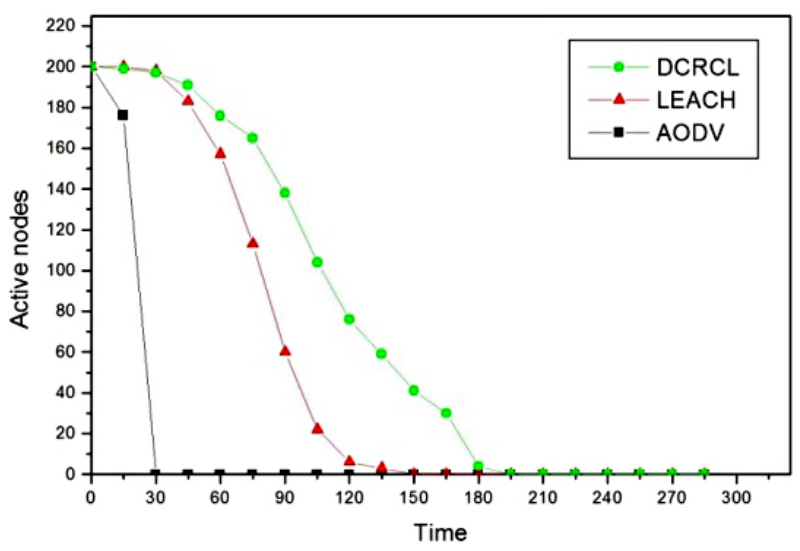

(a)

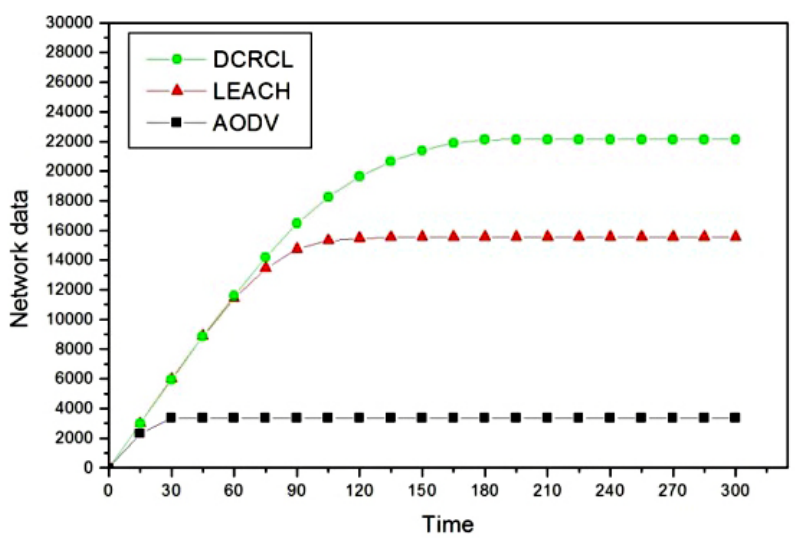

(b)

Figure 4. (a) Active Nodes; (b) Data Received by BS. 
Considering the generalization, more experiments are performed by using different number of nodes from 50 to 500. It is shown in Figure5 that the proposed algorithm in this paper also performed well for large scale networks. Figure 5(a) shows that average life cycle of DCRCL is 30.7 percent higher than $\mathrm{LEACH}$ and the curve is smoother than LEACH which means our method works more steadily. In Figure 5(b), the average data received by BS in DCRCL is 29.8 percent higher than LEACH, and get to the highest 47.6 percent when the node number is 450 .

\section{Conclusion}

In this paper, a dynamic clustering routing algorithm considering node load for wireless sensor networks is presented. In which, CHs are selected by considering residual energy and node load, a cost function based on energy and distance is used to set up the cluster and CHs use multi-hop inter-cluster routing model which is established by the attribute equilibrium function. The experiment results show that the proposed algorithm is more efficient with respect to network life cycle and network data than LEACH and AODV.

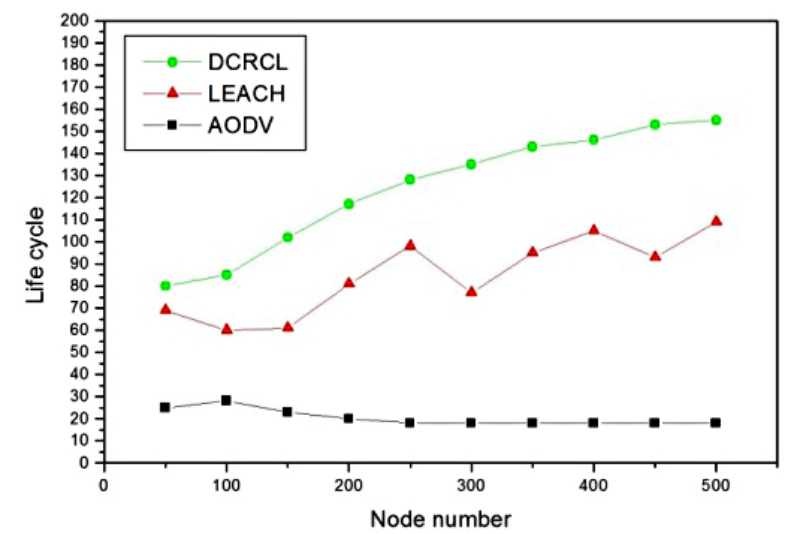

(a)

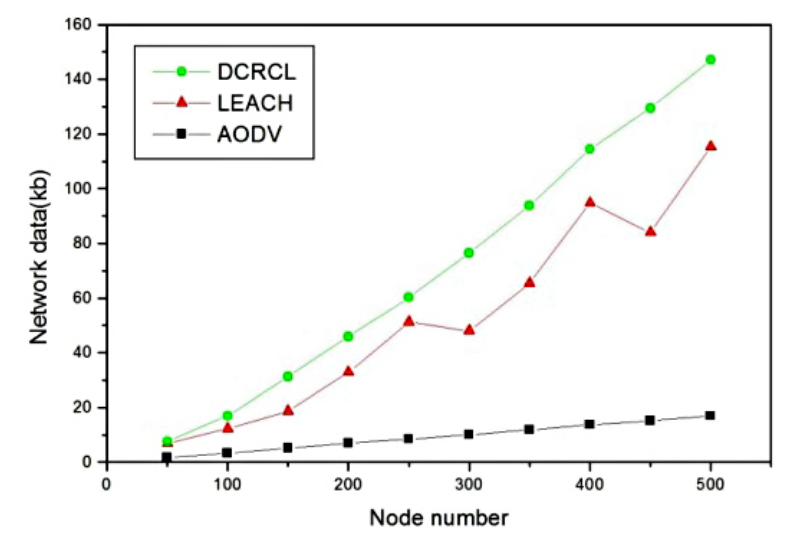

(b)

Figure 5. (a) Life cycle; (b) Date Received by BS. Comparison between DCRCL, LEACH and AODV for different node number.

\section{REFERENCES}

[1] T. F. Akyildiz, W. Su, Y. Sankarasubramaniam and E. Cayirci, "Wireless Sensor Networks: A Survey," Computer Networks, Vol. 38, No. 4, 2002, pp. 393-422.

[2] G. Gupta and M. Younis, "Load-Balanced Clustering of Wireless Sensor Networks,” IEEE International Conference on Communications, Vol. 3, 2003, pp. 1848-1852.

[3] P. Kuila and P. K. Jana, “An Energy Balanced Distributed Clustering and Routing Algorithm for Wireless Sensor Networks," IEEE International Conference on Parallel, Distributed and Grid Computing, 2012, pp. 200-225.

[4] J. G. Yu, "A Cluster-Based Routing Protocol for Wireless Sensor Networks with Non Uniform Node Distribution," Journal of Electronics and Communications, Vol. 66, 2012, pp. 54-61.

[5] W. B. Heinzelman, A. P. Chandrakasan and H. Balakrishnan, "An Application Specific Protocol Architecture for Wireless Microsensor Networks,” IEEE Transactions on Wireless Communications, Vol. 1, No. 4, 2002, pp. 660-670. http://dx.doi.org/10.1109/TWC.2002.804190

[6] S. Lindsey and C. S. Raghavendra, "PEGASIS: Power Efficient Gathering in Sensor Information Systems," IEEE Transactions on Parallel and Distributed Systems, Vol. 13, No. 9, 2002, pp. 924-930.

http://dx.doi.org/10.1109/TPDS.2002.1036066

[7] O. Younis and S. Fahmy, "HEED: A Hybrid, Energy-Efficient, Distributed Clustering Approach for Ad Hoc Sensor Networks," IEEE Transactions on Mobile Computing, Vol. 3, 2004, pp. 366-379. http://dx.doi.org/10.1109/TMC.2004.41

[8] B. C. Gong, L. Y. Li, S. R. Wang and X. J. Zhou, "Multihop Routing Protocol with Unequal Clustering for Wireless Sensor Networks," Colloquium on Computing, Communication, Control, and Management, Vol. 2, 2008, pp. 552-556.

[9] P. Kuila and P. K. lana, "Improved Load Balanced Clustering Algorithm for Wireless Sensor Networks,” Proceedings of the 2011 International Conference on Advanced Computing, Networking and Security, 2012, pp. 399-404.

[10] C. Petrioli, M. Nati, P. Casari, M. Zorzi and S. Basagni, "ALBA-R: Load-Balancing Geographic Routing Around Connectivity Holes in Wireless Sensor Networks," IEEE Transactions on Parallel and Distributed Systems, 2013, pp. 1-11. 